\title{
O efeito de um programa multiesportivo adaptado sobre a qualidade de vida de dependentes de crack em tratamento: um ensaio clínico randomizado
}

http://dx.doi.org/10.11606/1807-5509202000020345

\author{
Filipe Pitágoras Rodrigues MAGALHÃES* \\ Mariana Ferreira de SOUZA* \\ Terezinha Abel ALVES \\ Ferdinando de Oliveira CARVALHO* \\ J osé Fernando Vila Nova de MORAES* \\ *Universidade Federal \\ do Vale do São \\ Francisco, Petrolina \\ $\mathrm{PE}$, Brasil.
}

\section{Resumo}

0 presente estudo objetivou verificar o efeito de um programa multiesportivo adaptado sobre a qualidade de vida (QV), de dependentes de crack internados numa comunidade terapêutica (CT). A amostra compreendeu 17 pacientes divididos em dois grupos, onde o grupo intervenção $(G I), n=08$, participou de 10 sessões de treinamento e o grupo controle (GC), $n=09$, permaneceu desenvolvendo as atividades da CT. As sessões eram compostas por jogos cooperativos, corrida/caminhada em intensidade autoselecionada e xadrez motivacional e tinham duração de 90 minutos, três vezes por semana. Avaliou-se a QV utilizando o questionário WHOQOL-BREF. Empregou-se as análises de variância (ANOVA) mista para a comparação entre os momentos (pré vs. pós) e entre os grupos. 0 nível de significância adotado foi $p<0,05$ e 0 software utilizado para as análises foi o SPSS versão 22.0. Os resultados revelaram uma melhora significativa nos domínios meio-ambiente (pré $=12,37 \pm 1,91$ vs. pós $=14,67 \pm 2,38, p=0,03$ ) e autoavaliação da $\mathrm{QV}$ (pré=14,22 $\pm 2,33$ vs. pós $=15,71 \pm 2,43, p=0,01)$ no $\mathrm{Gl}$. Os resultados são promissores por demonstrarem a viabilidade de um programa multiesportivo nesta população e por auxiliar significativamente na melhoria de domínios da QV.

Palavras-chave: Exercício físico; Xadrez motivacional; Dependência química; Qualidade de vida.

\section{Introdução}

De acordo com a Organização Mundial de Saúde $(\mathrm{OMS})^{1}$, novas substâncias psicoativas, novos padrōes de consumo e associaçóes entre diferentes drogas tem surgido nos últimos anos, e este contexto favorece o surgimento de transtornos psiquiátricos de difícil tratamento como a dependência de substâncias (DS). A DS pode ser definida como um transtorno psiquiátrico debilitante que se caracteriza por uma procura compulsiva pela droga, apesar das graves consequências adversas associadas ao seu uso abusivo² ${ }^{2}$

Toda essa dinâmica faz emergir divergências de opinióes em relaçấo ao tratamento para a DS. Isso se dá pela complexidade, uma vez que se caracteriza como uma doença multifacetada, seu elevado custo do tratamento, além de difícil acesso ao tratamento, escassez de medicamentos efetivos, baixa adesão aos programas terapêuticos, abordagens ineficazes. Ainda, em diversas situaçóes o tratamento focado exclusivamente no consumo de drogas, e não na qualidade de vida do paciente, questóes que tornam ainda mais difícil o tratamento da $\mathrm{DS}^{3}$.

Apesar dos avanços relativos as abordagens terapêuticas na DS, há ainda uma grande lacuna a ser preenchida, pois embora existam medicamentos com eficácia modesta para a dependência de álcool, opiáceos e tabaco, nenhuma medicaçáo, ou programa terapêutico tem se mostrado eficaz para o tratamento da dependência por cocaína e, por conseguinte, a dependência por seus derivados, como o rrack $^{4}$.

Conforme Ribeiro e LARANJeIra 5 a dependência do crack traz grandes impactos a saúde geral do indivíduo, relaçôes sociais, degradação do estilo de vida e aspectos cognitivos. A dependência do crack requer uma abordagem terapêutica complexa, multidisciplinar e duradoura, em que muitas vezes o dependente não tem acesso. 
Devido a precariedade de tratamentos eficazes diversos pesquisadores tem explorado outros métodos, incluindo intervençôes baseadas em exercício físico $^{6,7,8}$, jogos cooperativos ${ }^{9,10}$, e xadrez motivacional ${ }^{11}$. Outro ponto a ressaltar-se que vem ganhando mais atenção em relação a dependência química é a necessidade de intervenções capazes de estimular mudanças globais no sujeito visto toda a complexidade da DS. Deste modo observa-se que qualidade de vida dos usuários de crack e outras drogas, é um indicador de resultados terapêuticos que impactam no sucesso do tratamento, visto que quanto maior escores de determinados domínios da qualidade de vida menor é a chance de recaída e sintomas psiquiátricos ${ }^{12}$. A literatura aponta que esta população apresenta qualidade de vida prejudicada por conta do estilo de vida arriscado e desta forma programas de intervenção na qualidade de vida

\section{Método}

\section{Amostra e natureza da investigação}

O presente estudo tratou-se de um estudo clínico randomizado (ECR) de natureza quase experimental. A amostra compreendeu a participação de 17 sujeitos divididos aleatoriamente em dois grupos, por meio de sorteio simples, sendo: Grupo Intervenção, $(\mathrm{n}=08)$ que participou do programa multiesportivo adaptado e Grupo Controle, ( $n=09)$ que permaneceu desenvolvendo as atividades habituais da comunidade terapêutica.

Esta comunidade terapêutica presta serviço multidisciplinar de acolhimento a adultos do sexo masculino diagnosticados com dependência química há mais de 20 anos.

A instituição é devidamente regularizada pela Secretaria Nacional Sobre Drogas (SENAD), como também atende os critérios estabelecidos pela Agência Nacional de Vigilância Sanitária, de acordo com a Resolução RDC No 29, de 30 de junho de 2011. E dispóe de: assistente social, psicóloga, capelão, pedagoga, médica psiquiatra, técnico de enfermagem, assistente administrativo, e uma equipe de educadores sociais devidamente capacitados. Sendo a única na região que oferece aos residentes e seus familiares atividades de terapias de campo na área de agricultura, terapias de grupo, atividades educativas, cursos profissionalizantes e atividades esportivas. A instituição possui capacidade para atender, simultaneamente, até destes pacientes tem grande relevância para o sucesso no tratamento ${ }^{6,12}$.

Desta forma, um programa de intervenção baseado em atividades físicas e motoras difundidas pela grande área de conhecimento em Educação Física é de grande importância, por apresentar baixo custo, pouco risco, e boa aderência nessa população ${ }^{13}$. O exercício físico promove benefícios à saúde mental e física, auxiliando diferentes fases do processo de dependência, como no manejo do desejo compulsivo (craving), na manutenção da abstinência, sendo um fator de proteção para a recaída ${ }^{7,14,15}$. Além de promover melhora na QV de adictos em tratamento ${ }^{16}$. Deste modo, o presente estudo buscou investigar a viabilidade de um programa multiesportivo adaptado sobre a qualidade de vida (QV) de dependentes de crack e em recuperação numa comunidade terapêutica.

30 adultos, por um tempo máximo de internação de aproximadamente um ano, dividido em acolhimento, conscientização e reinserção social.

O programa foi conduzido por três profissionais de Educação Física, um com especialização em Saúde Mental e experiência de cinco anos no tratamento de DS em CT, uma profissional de Educação Física especialista em Xadrez e um Doutor em Educação Física responsável pela aplicação dos testes. O programa multiesportivo ocorreu num período de dois meses (Fevereiro e Março de 2017). Foram realizadas 10 sessôes, três vezes por semana, com duração média de 90 minutos. Foram analisados os dados pré e pós intervenção. $\mathrm{O}$ estudo foi submetido e aprovado pelo Comitê de Ética e Deontologia em Estudos e Pesquisas (CEDEP) da Universidade Federal do Vale do São Francisco (UNIVASF) sob o registro: CAAE 60495116.0.0000.5196.

Critérios de Inclusão: Ter idade entre 18 e 55 anos; ser do sexo masculino; preencher os critérios para dependência de cocaína (usada sob a forma do crack) pela Classificaçáo Internacional de Doenças (CID-10), sendo o crack sua droga de preferência; estar em abstinência há, pelo menos, 10 dias; não ter alguma alteração que prejudique o desempenho no preenchimento dos instrumentos e participação na intervenção; e, ter assinado o TCLE (termo de 
consentimento livre e esclarecido).

Critérios de exclusão: Não completar pelo menos $70 \%$ do programa de intervenção proposto; interromper a utilização de medicamento prescrito pela médica psiquiatra da comunidade (caso aplicável).

\section{Testes e medidas}

Para a coleta de dados, foram aplicados os seguintes instrumentos:

Questionário sócio demográfico: Foi aplicada inicialmente para caracterização da amostra, uma ficha de dados sócios demográficos elaborada para definir o perfil sócio demográfico da amostra estudada, identificando características importantes para melhor avaliar o sujeito e verificar dados referentes ao consumo de substâncias psicoativas o conforme MAURINA ${ }^{17}$.

Questionário de prontidão para a atividade física (PAR-Q): Foi utilizado para triagem do risco cardiovascular, na qual, se o voluntário respondesse negativamente a todas as perguntas, estariam aptos a participar do programa.

\section{Qualidade de vida (QV)}

Foi medida pelo instrumento Escala de Qualidade de Vida (World Health Organization - Quality of Life- WHOQOL) que consiste de um questionário da Organização Mundial de Saúde (OMS) que visa investigar a satisfação e bem estar do indivíduo através de 26 questóes referentes aos domínios relacionados às habilidades físicas, psicológicas, condiçóes sociais, do meio ambiente e qualidade de vida geral. Sendo quanto maior a pontuação, melhor o nível de satisfação com a qualidade de vida ${ }^{18}$.

\section{Descrição da intervenção experimental}

O programa multiesportivo baseou-se em três conteúdos, desta forma, todas as sessóes foram compostas por jogos cooperativos, exercício aeróbio e jogos de tabuleiro (xadrez motivacional).

Os conteúdos foram orientados de forma progressiva, respeitando, sobretudo a individualidade biológica de cada participante.

A parte inicial de cada sessão funcionava como o aquecimento através da vivência lúdica de jogos cooperativos com duração de (05-10min), a segunda parte da sessão a corrida ou caminhada numa intensidade autoselecionada com duração média de (30-45min) e a terceira parte da sessão consistiu na vivência do xadrez motivacional (30-45 min).

\section{J ogos cooperativos}

Abordou-se o conteúdo de jogos cooperativos por ser um instrumento que visa estimular a cooperação, integração, socialização, criatividade, imaginação, comunicação, expressão corporal e a motivação através de atividades que têm um caráter lúdico, permitindo aos participantes momentos de descontração e alegria, fatores importantes para o bem-estar do ser humano ${ }^{19}$. Essas práticas podem ser realizadas tanto com materiais de apoio como sem eles. Alguns dos jogos cooperativos foram estruturados com o auxílio de diversos materiais, outros não. Os materiais utilizados serão: balóes, colchonetes, folhas de jornal, aparelho de som e CDs. Os residentes participarão dos seguintes jogos cooperativos, obedecendo esta ordem:

a) Círculo “maluco" - todos de mãos dadas em círculo. Cada participante sugeria uma tarefa ao grupo e estes sem soltar as mãos executavam a tarefa.

b) Jogos com balóes - atividade realizada com variações. a) "Todos em suspensão" - cada participante deve portar um balão. O objetivo é mantê-lo em suspensão sem que para isso tenha que ficar segurando o mesmo. Com o passar do tempo outros balóes são colocados na atividade, aumentando assim a dificuldade. b) "Troca de passes em pequenos grupos" - os participantes são divididos em pequenos grupos. O objetivo de cada grupo é trocar passes com as mãos ou pés, sem deixá-los cair no chão. Inicia-se com três balóes, e, no decorrer da atividade, este número aumenta. c) "Sanduíche andante" - participantes são divididos em pequenos grupos. Cada grupo deve formar um "sanduíche" com balóes e, assim, devem locomover-se pelo espaço destinado sem deixar os balóes caírem no chão.

c) Alongamento muscular passivo em duplas - alongamentos musculares de membros inferiores. Inicialmente, um componente da dupla recebe auxílio para realizar a atividade. Em seguida, ocorre a troca. Por possuírem características cooperativas e não competitivas, os jogos cooperativos não apresentam pontuaçóes avaliativas de desempenho.

d) Pega cauda - cada participante recebia 
uma palha de coco que simbolizava uma cauda, colocada no short, e ao comando do professor todos tentavam pegar as caudas uns dos outros. $\mathrm{O}$ jogo terminava quando todos os participantes perdiam suas caudas.

e) Corrente humana - um participante era convidado para ser o pega, e os demais teriam que fugir dele no espaço determinado, quando tocados, os participantes se uniam ao pegador até formarem uma corrente humana gigante. $\mathrm{O}$ jogo terminava quando todos eram pegos e se uniam a corrente.

\section{Corrida ou caminhada com intensidade autoselecionada}

A corrida ou caminhada autoselecionada fez parte da intervenção por se tratar de um exercício de baixo custo, baixo-risco, e por ter um potencial não farmacológico muito importante como adjuvante no tratamento da dependência como ressalta $\mathrm{LYNCH}^{7}$, além de proporcionar um constructo motivacional por permitir que o sujeito escolha intensidade que deseja exercitar-se de forma confortável e prazerosa ${ }^{20,21}$.

O conteúdo corrida ou caminhada consistiu na segunda parte da sessão e teve duração média de 30-40 min, com intensidade autoselecionada, sendo sempre precedida por uma série de informaçóes sobre à autosseleção da intensidade fornecida de maneira individual aos participantes pelo responsável do estudo, seguindo as orientaçōes de DA Silva ${ }^{21}$. Resumidamente, as seguintes informaçóes foram repassadas: "Intensidade autosselecionada é caracterizada como a velocidade que você julga adequada para uma duração estipulada de atividade, no caso do presente estudo, 30 minutos de caminhada ou corrida. Por favor, nós gostaríamos que você selecionasse uma velocidade de caminhada que julgue preferida. Essa velocidade preferida de corrida ou caminhada deve ser aquela que considera apropriada para você".

\section{Xadrez motivacional}

O conteúdo xadrez motivacional, que consiste no jogo de xadrez associado à entrevista motivacional, foi vivenciado em grupo após a apresentaçáo inicial dos pesquisadores. As regras do jogo de xadrez foram explicadas aos participantes na primeira sessão. Posteriormente, foi entregue a cada participante uma folha com as regras escritas, para consulta em caso de dúvidas. A folha funcionou como auxílio externo de memória de trabalho.

A metodologia de trabalho do xadrez motivacional foi adaptada conforme GonÇALVEs $^{11}$. O grupo foi divido em duplas em cada aula revezando entre si sob a orientação do pesquisador. Em seguida, os participantes vivenciavam o xadrez motivacional durante 30 à $45 \mathrm{~min}$. Por fim, como parte final da aula ocorria um processo de mediaçáo embasado em estratégias motivacionais relacionadas ao xadrez por cerca 10 à 15 minutos.

As adaptaçóes de tempo de aplicaçáo do xadrez motivacional foram necessárias em virtude das demais tarefas realizadas pelos participantes no CT, como: limpeza do local, preparo de refeiçóes e auxílio nas obras realizadas no local.

\section{Análise estatística}

Foi realizada uma análise descritiva dos dados, expressos em média e desvio-padrão. A normalidade dos dados foi testada por meio do teste de Shapiro-Wilk. Para a comparação entre os grupos (intervenção e controle) e momentos (pré e pós), realizou-se uma Análise de Variância (ANOVA) mista. O teste-T de Student independente foi aplicado para comparação dos dados clínicos entre os grupos. O nível de significância adotado foi $p<0,05$ e o software utilizado para efetuar as análises foi o Statistical Package for the Social Sciences (SPSS), versão 22.0 para Windows. 


\section{Resultados}

A amostra foi composta por 17 participantes do sexo masculino, com média de idade de 35 anos, $52 \%$ possuía ensino fundamental incompleto, $83,4 \%$ estava internada por conta do crack, $76 \%$ recebia até um salário mínimo, a maior parte dos sujeitos se declararam solteiros $43,8 \%$ com renda mensal de até um salário mínimo. A TABELA 1 mostra as características sócio-demográficas dos participantes.

TABELA 1- Perfil dos participantes acolhidos na CT entre março e abril de 2017, n=17.

\begin{tabular}{|c|c|c|c|c|}
\hline \multirow{2}{*}{ Variável } & \multicolumn{2}{|c|}{ Intervençáo } & \multicolumn{2}{|c|}{ Controle } \\
\hline & Média (DP) & n (\%) & Média (DP) & n (\%) \\
\hline Tempo de intern. (dias) & $154,89(98,79)$ & & $157,50(90,35)$ & \\
\hline Idade (anos) & $32,22(8,79)$ & & $39,50(8,55)$ & \\
\hline \multicolumn{5}{|l|}{ Escolaridade } \\
\hline $1^{\circ}$ incompleto & & $4(44,4 \%)$ & & $5(62,5 \%)$ \\
\hline $1^{\circ}$ completo & & $3(33,3 \%)$ & & $1(12,5 \%)$ \\
\hline $2^{\circ}$ incompleto & & - & & $1(12,5 \%)$ \\
\hline $2^{\circ}$ completo & & $1(11,1 \%)$ & & - \\
\hline Superior incompleto & & $1(11,1 \%)$ & & $1(12,5 \%)$ \\
\hline \multicolumn{5}{|l|}{ Renda mensal } \\
\hline 01 salário & & $6(66,7 \%)$ & & $7(87,5 \%)$ \\
\hline 02 salários & & $2(22,2 \%)$ & & $1(12,5 \%)$ \\
\hline 03 salários & & $1(11,1 \%)$ & & - \\
\hline \multicolumn{5}{|l|}{ Moradia } \\
\hline Sozinho & & $1(11,1 \%)$ & & $3(37,5 \%)$ \\
\hline Pai e mãe & & $1(11,1 \%)$ & & - \\
\hline Pai ou mãe apenas & & $4(44,4 \%)$ & & - \\
\hline Pai, mãe e outros & & - & & $1(12,5 \%)$ \\
\hline Companheira apenas & & $1(11,1 \%)$ & & $1(12,5 \%)$ \\
\hline Mais duas & & $1(11,1 \%)$ & & $3(37,5 \%)$ \\
\hline \multicolumn{5}{|l|}{ Estado civil } \\
\hline Solteiro & & $3(33,3 \%)$ & & $4(50,0 \%)$ \\
\hline Casado & & $3(33,3 \%)$ & & $3(37,5 \%)$ \\
\hline Divorciado & & $1(11,1 \%)$ & & - \\
\hline Com companheira & & $1(11,1 \%)$ & & - \\
\hline Separado & & - & & $1(12,5 \%)$ \\
\hline \multicolumn{5}{|l|}{ Religiáo } \\
\hline Católica & & $2(22,2 \%)$ & & $2(25,0 \%)$ \\
\hline Evangélica & & $7(77,8 \%)$ & & $6(75,0 \%)$ \\
\hline \multicolumn{5}{|l|}{ Filhos } \\
\hline 01 filho & & $7(77,8 \%)$ & & $6(75,0 \%)$ \\
\hline 02 filhos & & - & & $1(12,5 \%)$ \\
\hline 03 filhos & & $1(11,1 \%)$ & & $1(12,5 \%)$ \\
\hline
\end{tabular}


Na TABELA 2 são apresentados os dados clínicos dos participantes da pesquisa. Por meio do teste-T de Student foi possível observar uma diferença estatisticamente significante apenas no tempo de uso da substância, onde o grupo controle apresentou média mais alta $(24,00 \pm 6,61$ vs. $15,11 \pm 9,28 ; \mathrm{p}=0,04)$.

TABELA 2 - Dados clínicos dos participantes, n=17.

${ }^{*} p<0,05$ para grupo intervenção.

\begin{tabular}{lcc}
\hline & Intervençáo & Controle \\
\hline Idade (anos) & $32,22 \pm 8,79$ & $39,50 \pm 8,55$ \\
Idade de início do uso (anos) & $17,11 \pm 5,08$ & $15,50 \pm 3,16$ \\
Anos de uso (anos) & $15,11 \pm 9,28$ & $24,00 \pm 6,61^{*}$ \\
Internações prévias (qtd.) & $1,56 \pm 0,88$ & $1,63 \pm 0,74$ \\
Tempo de internação (meses) & $154,67 \pm 98,87$ & $157,75 \pm 90,24$ \\
\hline
\end{tabular}

Três participantes apresentavam comorbidades no momento do estudo, sendo: depressão, transtorno de ansiedade e transtorno afetivo bipolar. Ainda, 50\% dos internos tomavam medicações prescritas pela médica psiquiatra da comunidade, como: Nortriptilina, Amitriptilina, Fluoxetina, Risperidona, Halol, Clonazepan, Diazepan, Fenergan e Biperideno.

A TABELA 3, por sua vez, traz os escores dos diferentes domínios de qualidade de vida de acordo com o grupo (controle ou intervenção) e o momento (pré e pós). Neste sentido, foi possível observar que houve um aumento significativo entre os momentos do grupo intervenção nos domínios meio ambiente e autoavaliação. No domínio meio-ambiente os escores aumentaram de 12,37 $\pm 1,91$ (pré) para 14,67 $\pm 2,38$ (pós), $\mathrm{p}=0,03$. Já no domínio autoavaliação, houve um incremento de $14,22 \pm 2,33$ (pré) para 15,71 $\pm 2,43$ (pós), $\mathrm{p}=0,01$. Ainda, pode-se observar uma diferença nos escores de autoavaliação, no momento pré, entre os grupos controle e intervenção, onde o grupo controle apresentou escores mais elevados $(17,00 \pm 2,10$ vs. $14,22 \pm 2,33 ; \mathrm{p}=0,01)$.

TABELA 1 - Perfil dos participantes acolhidos na CT entre março e abril de 2017, n=17.

\begin{tabular}{lccccc}
\hline & Intervenção & Controle & Efeitos & F & $P$ \\
\hline Dom. Físico & & & & & \\
Pré & $14,41 \pm 3,66$ & $16,09 \pm 1,59$ & Grupos & 0,84 & 0,37 \\
Pós & $16,11 \pm 2,18$ & $16,21 \pm 1,86$ & Tempo & 1,13 & 0,31 \\
$\Delta$ & 1,70 & 0,12 & Interação & 0,47 & 0,51 \\
Tam. do Efeito & $-0,58$ & $-0,06$ & & &
\end{tabular}

Dom. Psicol.

${ }^{*} \mathrm{p}<0,05$ para pré no mesmo grupo.

$\dagger p<0,05$ para pré no grupo intervenção.

Pré
Pós
$\boldsymbol{\Delta}$
Tam. do Efeito

Dom. R. Sociais

Pré

$\Delta$

Tam. do Efeito

Pré

$\begin{array}{cc}14,25 \pm 3,39 & 13,91 \pm 6,88 \\ 16,09 \pm 1,30 & 16,00 \pm 1,33 \\ 1,84 & 2,09 \\ -0,78 & -0,51\end{array}$

\section{Grupos}

0,01

0,91

Tempo

2,24

0,16

Interação

0,02

0,89

$13,78 \pm 3,20$
$16,57 \pm 4,80$
2,79

$16,44 \pm 1,82$

Grupos

0,64

0,44

$15,83 \pm 1,11$

Tempo

1,57

0,23

$-0,69$

$-0,61$

Interação

4,24

0,06

0,41 
Continuação

Dom. Meio Amb.

$\begin{array}{lccccc}\text { Pré } & 12,37 \pm 1,91 & 13,83 \pm 1,72 & \text { Grupos } & 0,79 & 0,39 \\ \text { Pós } & 14,67 \pm 2,38^{*} & 14,23 \pm 1,02 & \text { Tempo } & 5,69 & 0,03 \\ \Delta & 2,30 & 0,40 & \text { Interação } & 3,97 & 0,07 \\ \text { Tam. do Efeito } & -1,07 & -0,29 & & & \\ \text { Dom. AutoAv. } & & & & \\ \text { Pré } & 14,22 \pm 2,33 & 17,00 \pm 2,10 \dagger & \text { Grupos } & 1,48 & 0,24 \\ \text { Pós } & 15,71 \pm 2,43^{*} & 15,75 \pm 1,98 & \text { Tempo } & 0,35 & 0,56 \\ \Delta & 1,49 & -1,25 & \text { Interação } & 8,11 & 0,01 \\ \text { Tam. do Efeito } & -0,62 & 0,61 & & & \\ \text { Dom. Total } & 13,69 \pm 2,40 & 15,65 \pm 0,74 & \text { Grupos } & 2,01 & 0,18 \\ \text { Pré } & 15,72 \pm 1,55 & 15,47 \pm 0,83 & \text { Tempo } & 2,95 & 0,11 \\ \text { Pós } & 2,03 & -0,18 & \text { Interação } & 4,46 & 0,06 \\ \Delta & -1,02 & 0,23 & & & \end{array}$

\section{Discussão}

Este estudo objetivou investigar o efeito e a viabilidade de um programa multiesportivo adaptado (atividades físico-cognitivas) sobre a qualidade de vida de dependentes de crack em tratamento.

Os resultados aqui apresentados se assemelham aos do último levantamento nacional sobre o perfil do usuário de crack no Brasil realizado por Bastos e Bertoni22, na qual a média de idade dos participantes da pesquisa era de 30 anos, com baixa escolaridade, predominantemente solteiros $(60,64 \%)$ e classificados como baixa renda com renda mensal de até 01 salário mínimo.

Os principais achados deste estudo foram que um programa multiesportivo composto por jogos cooperativos, corrida e caminhada com intensidade autoselecionada e xadrez motivacional promoveu a melhora na qualidade de vida, especificamente nos domínios meio ambiente e autoavaliação da qualidade de vida por parte do grupo que recebeu a intervenção. O programa multiesportivo também se mostrou viável no contexto da comunidade terapêutica, visto que não houve desistência de nenhum dos oito participantes do GI após o início do programa, além de 75\% de frequência, fato muito difícil de ser alcançado devido à dificuldade que é trabalhar com esta população.

$\mathrm{O}$ presente estudo se difere dos demais por ter uma intervenção baseada em princípios fundamentais para o que é preconizado para tratamento da DS atualmente, onde busca-se desenvolver aspectos relacionados a motivação, reabilitação cognitiva, qualidade de vida, e aptidão física de forma conjunta ${ }^{3,14}$. Ademais, vale ressaltar que a maioria dos estudos acerca do exercício e tratamento da dependência de psicoestimulantes foram realizados com ratos e este estudo busca testar estas hipóteses com seres humanos, comparando um grupo intervenção e um grupo controle por meio de um ensaio clínico randomizado. Ainda, se propóe a ser o primeiro estudo a aliar na sua intervenção jogos cooperativos, corrida autosselecionada e xadrez motivacional numa mesma sessão, possibilitando aos pacientes a ampliação do repertório motor, cognitivo, socialização e bem estar.

No que tange a qualidade de vida dos participantes da pesquisa observou-se diferenças estatisticamente significativas no domínio meio ambiente e autoavaliação da qualidade de vida, conquanto, no domínio meio ambiente houve diferença significativa entre os momentos no grupo intervenção $p<0,05$, como mostra a TABELA2. No que tange ao domínio autoavaliação da qualidade de vida houve diferença significativa entre o momento pré dos grupos além dos momentos no grupo intervenção $p<0,05$. Nesse sentido, o maior 
escore apresentado pelo GC pré-intervenção em comparação com o GI pode ter ocorrido em razão da média de tempo no tratamento dos participantes do GC ser maior.

$\mathrm{Na}$ avaliação da QV dos dependentes químicos através do WHOQOL-Bref, o Domínio Ambiental obteve melhora significativa. Tendo em vista que esse domínio pode ser compreendido por meio de aspectos envolvendo o ambiente no lar, recursos financeiros, cuidados de saúde e sociais - disponibilidade e qualidade, oportunidades de adquirir novas informaçóes e habilidades, participação e oportunidades de recreação/ lazer, ambiente físico (poluição/ruído/trânsito/clima) e transporte. Este domínio foi influenciado pelo programa multiesportivo onde proporcionou aos participantes oportunidades de lazer, cuidados de saúde, proporcionou novas informaçóes e treinamento de novas habilidades.

Em pesquisa realizada por BARBANTI ${ }^{16}$ utilizando o SF-36 para avaliar a QV de dependentes químicos pré e pós intervenção de dois meses e quatro meses com um programa de exercícios que envolvia: yoga, karatê, musculação, ginástica localizada, condicionamento físico, caminhada e alongamento, verificou-se que houve melhora na qualidade de vida dos indivíduos que realizaram atividades físicas após dois meses e após quatro meses do início do tratamento.

Em estudo realizado por Muller ${ }^{23}$ com 35 dependentes de múltiplas drogas, verificou-se uma melhora significativa no domínio de saúde física dos participantes que finalizaram o programa de exercícios $(\mathrm{F}(1,26)=9,532 ; \mathrm{p}=0,005)$, saúde psicológica $(\mathrm{F}$ $(1,26)=5,813 ; \mathrm{p}=0,023)$, conquanto no domínio (relaçôes sociais e meio ambiente), não houve interações estatisticamente significativas, tampouco no domínio ambiental $(F(1,26)=0,913 ; p=0,348)$. Nesse sentido, os dados relativos ao domínio relaçóes sociais se assemelham aos nossos resultados, contudo, difere-se quando comparados ao domínio ambiental em que no presente estudo observaram-se melhoras significativas.

GimÉNEZ-MeSEGUER et al. ${ }^{24}$ avaliaram as alteraçōes de qualidade de vida em 47 pacientes dependentes de drogas, onde 25 participaram da intervenção por meio de um programa de exercícios em grupo e 22 do controle participando da rotina habitual da clínica. Foi avaliado a qualidade de vida por meio do instrumento (SF-36) pré e pós intervenção com um programa de atividades físicas monitorada de 12 semanas. Os resultados mostraram melhorias após a intervenção nos domínios Função Física percebida $(\mathrm{F}(1,35)=$ 8,72; $\mathrm{p}<0,01)$, Saúde Geral ( $\mathrm{F}(1,35)=4,16 ; \mathrm{p}<0,05$; Vitalidade $(\mathrm{F}(1,35)=17,00 ; \mathrm{p}<0,001)$, Funçâo
Social $(\mathrm{F}(1,35)=15,74 ; \mathrm{p}<0,001)$ e Saúde Mental $(\mathrm{F}(1,35)=14,74 ; \mathrm{p}<0,001 ;)$, em comparação com o grupo controle.

Em outro estudo com dependentes em tratamento, RoessLE ${ }^{25}$, que se concentrou nos efeitos do exercício na melhoria da consciência corporal e da autoconfiança. Os achados indicaram aumento vitalidade para realizar atividades de vida diária e melhorias na dimensão social. Os resultados deste estudo fornecem informaçóes sobre a importância do exercício para a qualidade de vida e o processo de recuperaçáo de pacientes dependentes de drogas. Estes resultados corroboram com os dados do presente estudo.

A literatura afirma que a qualidade de vida dos usuários de crack e outras drogas, é um indicador de resultados terapêuticos que impactam no sucesso do tratamento, visto que quanto maior escores de determinados domínios da qualidade de vida menor é a chance de recaída e sintomas psiquiátricos ${ }^{12}$. Deste modo se faz necessário maior atenção em aspectos relacionados aos domínios da QV, e abordagens terapêuticas que busquem melhorá-los visto a sua importância. Corroborando com essa afirmativa em pesquisa recente realizada por McQuAID et al. ${ }^{26}$ com 855 pacientes canadenses DS em tratamento por meio do Survey of life in recovery from addiction in Canada, 69,1\% da amostra avaliada destacou a melhora da qualidade de vida como o primeiro fator mais importante ao iniciar a recuperação. Deste modo o presente estudo apresenta grande relevância visto que a intervenção produziu melhoras em domínios da qualidade de vida, aspecto esse de suma importância para a recuperaçáo e melhores resultados no tratamento.

Apesar de o presente estudo trazer informaçóes importantes acerca da intervenção com dependentes de crack, ele possui limitaçóes. Inicialmente, a quantidade de participantes não permite extrapolar o resultado em larga escala. Ainda, houve uma pequena perda amostral ao longo da coleta, visto que um participante concluiu o programa do CT e, em virtude das tarefas desempenhadas no centro, alguns voluntários não conseguiram participar de todas as avaliaçóes.

Adicionalmente, é importante ressaltar que os participantes do grupo controle, apesar de náo participarem das intervençôes do grupo experimental, realizavam as atividades programadas do $\mathrm{CT}$, que também envolviam a prática de atividade física (futebol). Isto pode ter influenciado na melhora da aptidão cardiorrespiratória deles.

Por fim, em virtude do uso de um programa multiesportivo, envolvendo jogos cooperativos, atividade física e xadrez motivação, é impossível saber 
qual intervenção efetivamente impactou a qualidade de vida dos participantes. Ainda, outros fatores que poderiam impactar a percepção da qualidade de vida, como o aspecto alimentar, por exemplo, não foi levado em consideração no presente estudo. Entretanto, é válido ressaltar que os indivíduos de ambos os grupos (controle e intervenção) realizavam as mesmas refeiçôes.

Diante do exposto, conclui-se que 10 sessóes multiesportivas foram eficazes no que ser refere ao incremento nos domínios meio-ambiente e autoavaliação da qualidade de vida da população estudada. Estes resultados são promissores por demonstrarem a viabilidade do programa multiesportivo durante o tratamento da dependência química numa comunidade terapêutica.

Este trabalho traz uma nova perspectiva no que tange a prescrição do exercício físico para pessoas em tratamento da DS, ele apresenta uma proposta dinâmica de atividades motoras evidenciadas pela literatura que até então eram utilizadas separadamente. $\mathrm{O}$ presente estudo expóe a necessidade do melhor planejamento de atividades motoras ou esportivas mais abrangentes para esta população alertando para a necessidade da implementaçáo de práticas corporais desportivas capazes de promover melhoras na função executiva, além de melhoras cognitivas, fisiológicas relacionadas à qualidade de vida e necessidades específicas do dependente de crack e outras drogas. Este programa buscou responder a uma lacuna na literatura que é a sistematização de programas específicos e metodologias capazes de fazerem parte de um tratamento complexo de longo prazo, na qual demanda estímulos diversificados a fim de favorecer a adesão e aderência no programa terapêutico. No entanto mais pesquisas precisam ser realizadas, sobretudo, desenvolvendo intervençóes com pacientes no início do tratamento.

\title{
Conflito de interesses
}

Os autores declaram que não há conflito de interesses.

\section{Agradecimentos}

Programa de Pós-Graduação em Educação Física (PPGEF/UNIVASF) e Grupo de Estudos e Pesquisa em Genética e Exercício (GEPEGENE).

\begin{abstract}
Effect of an adapted multisport program on quality of life of crack dependents under treatment: a randomized clinical trial

The present study aimed to verify the outcome of an adapted multi-sport program over the quality of life (OL) of crack dependents admitted to a therapeutic community (TC). The sample consisted of 17 patients divided into two groups, where the intervention group (IG), $n=08$, participated in 10 training sessions and the control group (CG), $n=09$, continued to develop as CT activities. The sessions were composed of cooperative games, self-selected intensity / walk or run and motivational chess and had duration of 90 minutes, three times a week. OL was assessed using the WHOOOL-BREF questionnaire. A mixed analysis of variance (ANOVA) was performed to compare moments (pre vs. post) and groups. The significance level adopted was $p<0.05$ and the software used was the SPSS version 22.0. The results showed a significant improvement in the environmental domains ( $p r e=12.37 \pm 1.91$ vs. post $=14.67 \pm 2.38, p=0.03$ ) and selfassessment of $\mathrm{OL}$ ( $p r e=14.22 \pm 2.33$ vs. post $=15.71 \pm 2.43, \mathrm{p}=0.01$ ) in the IG. The results are promising since they demonstrate the viability of a multi-sport program and show an improvement of the domains of $\mathrm{QL}$ in this population.
\end{abstract}

KeYwords: Physical Exercise; Motivational Chess; Chemical Dependency; Quality of Life. 


\section{Referências}

1. World Health Organization WHO Expert Committee on Drug Dependence. Thirty-sixth report. World Health Organ Tech Rep Ser. 2015;(991):1-50.

2. Peter E. Nathan MC, Anne HS. History of the Concept of Addiction. Annual Review Of Clinical Psychology. 2016; 12:29-51.

3. Andrade ALM, De Micheli D. Innovations in the Treatment of Substance Addiction. Switzerland: Springer International Publishing. 2016.

4. Kim JH, Lawrence AJ. Drugs currently in Phase II clinical trial for cocaine addiction. Exp Opin Invest Drugs. 2014; 23(8):1105-1122.

5. Ribeiro M, Laranjeira, R. O tratamento do usuário de crack. 2a . ed. Porto Alegre: Artmed, 2012.

6. Da Costa, KG. Barbieri, JF. Hohl, R. Costa EC. Fontes, EB. Exercise training improves cardiorespiratory fitness and cognitive function in individuals with substance use disorders: a pilot study. 2016. Sport Sciences for Health, 1-5.

7. Lynch, W. J., Peterson, A. B., Sanchez, V., Abel, J., \& Smith, M. A. Exercise as a novel treatment for drug addiction: a neurobiological and stage-dependent hypothesis. Neuroscience \& Biobehavioral Reviews. 2013. 37(8), 1622-1644.

8. Ferreira SE. Dos Santos AKD. Okano AH., Gonçalves BDSB. Araújo JF. Efeitos agudos do exercício físico no tratamento da dependência química. Revista Brasileira de Ciências do Esporte. 2017; 39(2), 123-131.

9. Alves GSL, Araujo RB. A utilização dos jogos cooperativos no tratamento de dependentes de crack internados em uma unidade de desintoxicação. Rev Bras Med Esporte. 2012;18(2):77-80.

10. Braga JEF, Chaves NG, Lima AB, Oliveira RQ, Alves RS, Farias JA. Jogos cooperativos e relaxamento respiratório: Efeito sobre o craving e ansiedades. Rev Bras Med Esporte. 2016.

11. Goncalves PD., Ometto M. Bechara A. Malbergier A. Amaral R. Nicastri S. Andrade AG. Motivational Interviewing combined with chess accelerates improvement in executive functions in cocaine dependent patients: A one-month prospective study. Drug and alcohol dependence. 2014; 141, 79-84.

12. Paiva CB, Ferreira IB, Bosa VL, Narvaez JCM. Depression, anxiety, hopelessness and quality of life in users of cocaine /crack under outpatient treatment. Trends Psychiatry Psychother. 2017;39(1):34-42.

13. Abrantes AM, Battle CL, Strong DR, . Exercise preferences of patients in substance abuse treatment. Mental Health Phys Activ 2011;4:79-87.

14. Weinstock J. Weinstock MR. Farney NM. Elrod CE. Henderson EP. Weiss Exercise as a adjunctive treatment for substance use disorders: Rationale and intervention description. Journal of Substance Abuse Treatment, 72C (2017), pp. $40-47$.

15. Bardo MT, Compton WM. Does physical activity protect against drug abuse vulnerability? Drug Alcohol Depend. 2015.

16. Barbanti EJ. Efeito da atividade física na qualidade de vida em pacientes com depressão e dependência química. Revista Brasileira de Atividade Física \& Saúde. 2012; 11(1), 37-45.

17. Maurina Coelho RL. Habilidades sociais e de enfrentamento no tratamento de usuários de crack. - Porto. Alegre, 2016. $135 \mathrm{f}$. Tese (Doutorado)

18. Fleck MPA, et al. Application of the portuguese version of the abbreviated instrument of quality life WHOQOL-bref. Rev Saude Publica. 2000;34(2):178-83

19. Soler, R. Brincando e aprendendo com os jogos cooperativos. Rio de Janeiro: Sprint, 2008.

20. Ekkekakis P, Lind E, Vazou S. Affective responses to increasing levels of exercise intensity in normal-weight, overweight, and obese middle-aged women. Obesity. 2009.

21. Dasilva SG, Guidetti L, Buzzachera CF, Elsangedy F, Krinski, K, De Campos W, Baldari C. Psychophysiological responses to self-paced treadmill and overground exercise. 2011. Medicine \& Science in Sports \& Exercise, 43(6), 1114-1124.

22. Bastos FI , Bertoni N. Pesquisa nacional sobre o uso de cocaína crack - quem são crack e / ou usuários de cocaína fumantes similares no Brasil? Quantos deles vivem nas capitais brasileiras? Rio de Janeiro : ICICT / FIOCRUZ; 2014.

23. Muller AE, Clausen T. Group exercise to improve quality of life among substance use disorder patients. Scand J Public Health. 2015;43(2):6.

24. Giménez-Meseguer J, Tortosa-Martínez J., Remedios FV. Benefits of exercise for the quality of life of drug-dependent patients. Journal of psychoactive drugs. 2015; 47(5), 409-416.

25. Roessler KK. Exercise treatment for drug abuse-a Danish pilot study. Scand J Public Health 38. 2010;(6):664-669. 
26. McQuaid RJ, Malik A, Moussouni K, Baydack N, Stargardter M, Morrisey M. (2017). Life in Recovery from Addiction in Canada. Ottawa, Ont.: Canadian Centre on Substance Use and Addiction.

Filipe Pitágoras Rodrigues Magalhães Av. J osé de Sá Maniçoba, S/ N - Centro 56304-917 - Petrolina - PE - Brasil E-mail: filipepitagoras@hotmail.com
Submetido: 19/08/2017

1a revisão: 01/02/2018

2a revisão: 27/04/2018

Aceito: 13/06/2018 